\title{
METABOLIC SYNDROME AND OBESITY AS FACTORS OF GOOD PROCNOSTIC ONCOLOGY IN WOMEN IN POST-MENOPAUSE WITH RECENT DIAGNOSIS OF BREAST CANCER
}

Andre Hideo Motoki ${ }^{1}$, Daniel de Araújo Brito Buttros ${ }^{1}$, Heloisa Maria de Luca Vespoli', Eduardo Carvalho Pessoa ${ }^{1}$, Eliana Aguiar Petri Nahas ${ }^{1}$

${ }^{1}$ Faculdade de Medicina de Botucatu - Botucatu (SP), Brazil.

Objective: To evaluate the association between metabolic syndrome (MS), obesity, and central fat deposition with the immunohistochemical profile (IHC) of breast cancer (BC) in postmenopausal women. Methods: A comparative cross-sectional clinical study was carried out with 63 women with recent BC and MS, compared to 126 women with recent BC, without MS (control group). Inclusion criteria were: women aged 45-75 years, amenorrhea $>12$ months, without previous cancer treatment, attended at a University Hospital. The groups were matched for age, time since menopause, and body mass index (BMI), in the proportion of 1 case for 2 controls, according to the sample calculation of at least 186 women in their entirety. Clinical and anthropometric data were collected; tumor size and grade and the IHC profile (ER, PR, HER2, and Ki67). By IHC convention, tumors were grouped into five subtypes: Luminal A (ER+, PR+, HER-2 -, and Ki-67 <14\%); Luminal B HER-2 - (ER+, PR+ or -, HER-2 -, and Ki-67 14\%); Luminal B HER-2+ (ER+, PR+ or -, HER-2+, and any Ki-67); Nonluminal HER-2 (ER-, PR-, HER2+, and any Ki-67); and Triple-negative (ER-, PR-, HER2-, and any Ki-67). Women with three or more diagnostic criteria were considered with MS: waist circumference (WC) $>88 \mathrm{~cm}$; TG $\geq 150 \mathrm{mg} / \mathrm{dL}$; HDL cholesterol<50 mg/dL; blood pressure $\geq 130 / 85 \mathrm{mmHg}$; glucose $\geq 100 \mathrm{mg} / \mathrm{dL}$. For statistical analysis, the Student's $t$-test, Gamma Distribution, $\mathbb{Z}^{2}$ test and logistic regression (odds ratio-OR) were used. Results: Among the participating women, the mean age, time since menopause and BMI were: $59.0 \pm 10.6$ years, $11.4 \pm 9.6$ years, and $28.5 \pm 5.5 \mathrm{~kg} / \mathrm{m}^{2}$, respectively; there was no statistical difference in the comparison between the groups. Women with MS had a higher occurrence of tumors $\leq 2 \mathrm{~cm}$ when compared to those without MS (49.2 vs. 31.8\%, respectively) ( $\mathrm{p}=0.038)$. Women with MS had a higher incidence of tumors with PR-positive ( $\mathrm{p}=0.046)$, HER2-negative ( $\mathrm{p}=0.038)$, when compared to women without MS (79.4 vs. $65.8 \%$ and 44.5 vs. $27.8 \%$, respectively). In obese patients $\left(B M I \geq 30 \mathrm{~kg} / \mathrm{m}^{2}\right)$, a higher proportion of HER2 negative tumors ( $\left.\mathrm{p}=0.047\right)$ was observed when compared to non-obese women (43.9 vs. 27.7\%, respectively). In the multivariate analysis, a higher risk for tumors of the Luminal B HER-2 negative subtype was observed among women with MS (OR 2.00, 95\%CI 1.03-3.89), obese (OR 2.03, 95\%CI 1.06-3.90), and with central deposition of fat (OR 1.96, 95\%CI 1.01-4.03). Conclusion: Metabolic syndrome, obesity, and central fat deposition correlate with factors of good prognosis for breast cancer, such as tumors $\leq 2 \mathrm{~cm}$, PR+ and HER2-, in postmenopausal women. 